(1)

CrossMark

\title{
Lipids and ketones dominate metabolism at the expense of glucose control in pulmonary arterial hypertension: a hyperglycaemic clamp and metabolomics study
}

\author{
Jacob T. Mey (10 ${ }^{1,2}$, Adithya Hari², Christopher L. Axelrod ${ }^{1,2,3}$, Ciarán E. Fealy ${ }^{2,4,5}$ \\ Melissa L. Erickson ${ }^{1,2}$, John P. Kirwan ${ }^{1,2,5}$, Raed A. Dweik ${ }^{2,6}$ and Gustavo A. Heresi ${ }^{2,6}$ \\ Affiliations: ${ }^{1}$ Integrative Physiology and Molecular Medicine Laboratory, Pennington Biomedical Research Center, \\ Baton Rouge, LA, USA. ${ }^{2}$ Inflammation and Immunity, Lerner Research Institute, Cleveland Clinic, Cleveland, \\ $\mathrm{OH}$, USA. ${ }^{3}$ Dept of Translational Services, Pennington Biomedical Research Center, Baton Rouge, LA, USA. \\ ${ }^{4}$ Diabetes and Metabolism Research Group, Maastricht University, Maastricht, The Netherlands. ${ }^{5}$ Metabolic \\ Translational Research Center, Endocrinology and Metabolism Institute, Cleveland Clinic, Cleveland, OH, USA. \\ ${ }^{6}$ Dept of Pulmonary Care and Critical Medicine, Respiratory Institute, Cleveland Clinic, Cleveland, OH, USA.
}

Correspondence: Gustavo A. Heresi, Respiratory Institute, Cleveland Clinic, Cleveland, $\mathrm{OH}$ 44195, USA. E-mail: heresigaccf.org

@ERSpublications

Highly technical metabolic approaches show that fasting nutrient metabolism in pulmonary arterial hypertension favours lipid and ketone metabolism and that, in response to hyperglycaemia, pancreatic 及-cell function is similar to control participants http://bit.ly/2uihG2Q

Cite this article as: Mey JT, Hari A, Axelrod CL, et al. Lipids and ketones dominate metabolism at the expense of glucose control in pulmonary arterial hypertension: a hyperglycaemic clamp and metabolomics study. Eur Respir J 2020; 55: 1901700 [https://doi.org/10.1183/13993003.01700-2019].

ABSTRACT Individuals with idiopathic pulmonary arterial hypertension (PAH) display reduced oral glucose tolerance. This may involve defects in pancreatic function or insulin sensitivity but this hypothesis has not been tested; moreover, fasting nutrient metabolism remains poorly described in PAH. Thus, we aimed to characterise fasting nutrient metabolism and investigated the metabolic response to hyperglycaemia in PAH.

12 participants (six PAH, six controls) were administered a hyperglycaemic clamp, while 52 (21 PAH, 31 controls) underwent plasma metabolomic analysis. Glucose, insulin, C-peptide, free fatty acids and acylcarnitines were assessed from the clamp. Plasma metabolomics was conducted on fasting plasma samples.

The clamp verified a reduced insulin response to hyperglycaemia in PAH ( $-53 \%$ versus control), but with similar pancreatic insulin secretion. Skeletal muscle insulin sensitivity was unexpectedly greater in PAH. Hepatic insulin extraction was elevated in $\mathrm{PAH}(+11 \%$ versus control). Plasma metabolomics identified 862 metabolites: 213 elevated, 145 reduced in PAH $(\mathrm{p}<0.05)$. In both clamp and metabolomic cohorts, lipid oxidation and ketones were elevated in PAH. Insulin sensitivity, fatty acids, acylcarnitines and ketones correlated with PAH severity, while hepatic extraction and fatty acid:ketone ratio correlated with longer six-min walk distance.

Poor glucose control in PAH could not be explained by pancreatic $\beta$-cell function or skeletal muscle insulin sensitivity. Instead, elevated hepatic insulin extraction emerged as an underlying factor. In agreement, nutrient metabolism in PAH favours lipid and ketone metabolism at the expense of glucose control. Future research should investigate the therapeutic potential of reinforcing lipid and ketone metabolism on clinical outcomes in PAH.

This article has an editorial commentary: https://doi.org/10.1183/13993003.00447-2020

This article has supplementary material available from erj.ersjournals.com

Received: 27 Aug 2019| Accepted after revision: 18 Jan 2020

Copyright OERS 2020 


\section{Introduction}

Pulmonary arterial hypertension (PAH) is a debilitating disease characterised by remodelling of the pulmonary vascular bed leading to elevated pulmonary arterial pressure requiring progressive compensation by the right ventricle, and culminates in right heart failure. Despite recent clinical advances, prognosis remains poor with estimated 3-year survival rates of $55-69 \%$ in newly diagnosed patients $[1,2]$. The pathobiology of PAH is incompletely understood and none of the currently available treatment modalities directly targets the underlying pulmonary vascular remodelling [3]. As such, there remains a dire need to identify the underlying pathophysiology of PAH to improve disease management.

Recent epidemiological observations have identified a disproportionate prevalence of obesity and diabetes comorbidities in individuals with $\mathrm{PAH}$; diseases hallmarked by insulin resistance and poor glycaemic control $[4,5]$. Corroborating data from in vitro, pre-clinical and biological experiments with humans have implicated metabolic disease in PAH pathogenesis [6]. We previously reported metabolic abnormalities in lipid [7] and carbohydrate metabolism [8,9] which were associated with poorer clinical outcomes. Importantly, patients with $\mathrm{PAH}$ exhibited overt glucose intolerance concomitant with reduced circulating insulin levels in response to an oral glucose tolerance test, suggesting impaired pancreatic $\beta$-cell function (i.e. reduced insulin secretion) in response to hyperglycaemia [9].

Additionally, ketone metabolism has been associated with the metabolic abnormalities in preclinical models of PAH [10] and humans [11]. Ketogenesis is primarily controlled by the liver in response to hormonal, sympathetic and nutritional input, which regulates circulating ketone concentrations, predominantly $\beta$-hydroxybutyrate $(\beta \mathrm{OHB})$. Another upstream regulator of $\beta \mathrm{OHB}$ is the delivery of lipids to the liver to be oxidised and subsequently converted into $\beta \mathrm{OHB}$. This regulation of $\beta \mathrm{OHB}$ is particularly relevant in $\mathrm{PAH}$ pathophysiology due to the characteristic progression of right heart failure, as the failing heart increasingly relies on $\beta \mathrm{OHB}$ for fuel [12]. Yet, plasma $\beta \mathrm{OHB}$ during fasting and in response to hyperglycaemia has not been characterised in PAH.

Further, methodologically rigorous approaches investigating metabolism are lacking in the PAH field. Poor oral glucose control has been reported in PAH [9, 13]; the underlying mechanisms remain to be defined, but may involve pancreatic $\beta$-cell function, skeletal muscle insulin sensitivity, ketogenesis or lipid metabolism. Thus, we utilised a combination of the state-of-the-art hyperglycaemic clamp procedure together with unbiased plasma metabolomics to assess $\beta$-cell function, insulin sensitivity and nutrient metabolism in two independent cohorts of PAH and Controls. We hypothesised that individuals with $\mathrm{PAH}$ would present with impaired pancreatic function and elevated ketone and lipid metabolism.

\section{Methods and materials \\ Subjects}

64 subjects were recruited as patients of the Cleveland Clinic's Respiratory Institute or from the greater Cleveland area (OH, USA); 12 received a hyperglycaemic clamp to assess pancreatic $\beta$-cell function, while 52 underwent untargeted metabolomics. PAH designation was based on pre-existing diagnosis [9]. This research was reviewed and approved by the Cleveland Clinic's Institutional Review Board and informed consent was obtained prior to initiating study procedures.

\section{Pancreatic $\boldsymbol{\beta}$-cell insulin secretion}

Participants reported to the Cleveland Clinic's Clinical Research Unit following stringent control procedures to minimise the influence of diet and physical activity on metabolic testing [14]. A hyperglycaemic clamp was performed in six PAH and six controls [15] to assess the pancreatic response to hyperglycaemia. Briefly, hyperglycaemia $\left(180 \mathrm{mg} \cdot \mathrm{dL}^{-1}\right)$ was rapidly achieved with a primed infusion of intravenous glucose (dextrose, 20\%) and maintained by variable rate infusion for $3 \mathrm{~h}$. Blood glucose was measured every $5 \mathrm{~min}$ (YSI 2300; STAT Plus, Yellow Springs, OH, USA) and the glucose infusion rate was adjusted according to the algorithm by DeFronzo et al. [16]. With hyperglycaemia, the plasma insulin response is well documented to be biphasic with an initial spike and nadir during the first $10 \mathrm{~min}$ (0-10 min, first phase) followed by a linear increase over time (10-180 min, second phase). During the first phase, blood was collected every $2 \mathrm{~min}$; during the second phase, blood was collected every $15 \mathrm{~min}$. Samples were processed and stored at $-80^{\circ} \mathrm{C}$ until analysis.

\section{Plasma analytes}

Insulin and C-peptide were assessed via radio-immunoassay (\#HI-14K and \#HCP-20K; Millipore Corporation, Billerica, MA, USA). The insulin:C-peptide molar ratio was calculated to assess hepatic insulin extraction. $\beta O H B$ was quantified via colorimetric assay (\#700190; Cayman Chemical, Ann Arbor, MI, USA). Free fatty acids (FFA) and acylcarnitines were quantified by liquid chromatography tandem mass spectrometry (LC/MS/MS). Untargeted metabolomic analysis (Metabolon Inc., Durham, NC, USA) 
was performed on fasting plasma samples. Additional details on the LC/MS/MS and metabolic approaches are available in the supplementary material.

\section{Glucose disposal and insulin sensitivity}

Glucose infusion rates (GIR) were calculated from the glucose required to maintain hyperglycaemia during steady-state (150-180 min) of the clamp. Insulin sensitivity was estimated as glucose metabolism $\left(\mathrm{M} ; \mathrm{mg} \cdot \mathrm{kg}^{-1} \cdot \mathrm{min}^{-1}\right)$ normalised to the prevailing insulin concentration $\left(\mathrm{M} / \mathrm{I} ; \mathrm{mg} \cdot \mathrm{kg}^{-1} \cdot \mathrm{min}^{-1} \cdot \mu \mathrm{U}^{-1} \cdot \mathrm{mL}^{-1}\right)$ during steady-state [15]. Indirect calorimetry was performed with an automated system ( $V_{\max }$ Encore, Viasys HealthCare, Yorba Linda, CA, USA) in a semi-darkened, thermoneutral $\left(22 \pm 1^{\circ} \mathrm{C}\right)$ environment.

\section{Statistical analysis}

Statistical analyses were performed using PRISM7 (GraphPad, La Jolla, CA, USA). Group differences were assessed by two-way repeated measures ANOVA (Group $\times$ Time). Post hoc multiple comparison tests were optimised for each analysis (detailed in figure legends). Area under the curve (AUC) was calculated using the trapezoidal method. AUC and baseline comparisons were evaluated by unpaired Student's t-test. Pearson's correlation assessed relationships among outcome measures of interest. Normality was determined by the Shapiro-Wilk test $(\alpha=0.05)$. Non-normal data were log-transformed. Data are expressed as mean \pm SD. Significance accepted at $\mathrm{p}<0.05$.

Metabolomic data are presented as fold-change and box-and-whiskers plots of scaled intensity values. Missing values were imputed with the minimum observed value for each compound. Following log-transformation, one-way ANOVA identified metabolites that differed between groups. To account for multiple comparisons, an estimate of the false discovery rate (q-value) was calculated. Significance accepted at $\mathrm{q}<0.1$.

\section{Results}

Hyperglycaemic clamp

Glucose, insulin and C-peptide

Participants (matched for age, BMI and sex) displayed similar fasting glucose, insulin and C-peptide concentrations (table 1). Basal metabolic rate was elevated in $\mathrm{PAH}$, when normalised for height, weight, age and sex, consistent with previous findings [9]. The insulin response to hyperglycaemia was reduced by $53 \%$ in $\mathrm{PAH}$ (figure 1b). C-peptide, a marker of pancreatic $\beta$ cell function and insulin secretion, was similar between groups (figure 1c). The glucose infusion rate required to maintain hyperglycaemia was similar for both groups (figure 1d). Insulin sensitivity (M/I) was elevated by $92 \%$ in PAH compared with controls (figure 1e).

\section{Ketogenesis $(\beta O H B)$ and hepatic insulin extraction}

Baseline $\beta \mathrm{OHB}$ was elevated in $\mathrm{PAH}(+40 \% ; \mathrm{p}=0.018)$ while both groups reached similar levels during hyperglycaemia (figure $2 \mathrm{a}$ ). Reductions in $\beta \mathrm{OHB}$ only reached significance in PAH (60 min, $120 \mathrm{~min}$, $180 \mathrm{~min}$; all $\mathrm{p}<0.02$ ), and $\beta \mathrm{OHB}$ remained greater in $\mathrm{PAH}$ throughout the clamp ( $\beta \mathrm{OHB}$ AUC, $\mathrm{PAH}$ : 13.0 $\pm 0.7 \mathrm{AU}$, control: $10.4 \pm 0.5 \mathrm{AU} ; \mathrm{p}=0.016$ ). Hepatic insulin extraction was greater in $\mathrm{PAH}$ during the first and second phase insulin response (figure $2 \mathrm{~b}$ and $\mathrm{c}$ ).

\section{FFAs and acylcarnitines}

Total FFAs were similar between groups at baseline and decreased equivalently throughout the clamp (figure 3a). Palmitate (C16:0) was the most prevalent lipid species, while palmitoleate (C16:1) was elevated in $\mathrm{PAH}$ at baseline (figure $3 \mathrm{~b}$ ). Acetylcarnitine was greater at baseline in $\mathrm{PAH}$ while both groups decreased with hyperglycaemia. The reduction was greater in $\mathrm{PAH}$ (delta, $\mathrm{PAH}:-6.3 \pm 0.4 \mu \mathrm{M}$, control: $-4.7 \pm 0.5 \mu \mathrm{M} ; \mathrm{p}=0.039$ ) (figure $3 \mathrm{c}$ ).

\section{Correlational analysis with metabolic measures}

Given these novel and unintuitive nutrient metabolism findings, we investigated relationships between the major metabolic pathways: insulin sensitivity, ketogenesis and hepatic extraction. Insulin sensitivity (M/I) correlated with hepatic extraction (figure $4 \mathrm{a}$ ) and $\beta \mathrm{OHB}$ throughout the clamp (figure $4 \mathrm{~b}$ ). These relationships remained significant when using first phase hepatic extraction or fasting $\beta \mathrm{OHB}$ as predictive factors for clamp-derived M/I (figure S1a and b). Furthermore, the ratio of fasting free fatty acids to ketones (FFA: $\beta \mathrm{OHB}$ ) predicted clamp-derived M/I when controlling for BMI and PAH status $\left(r^{2}=0.614\right.$, $p=0.045$; multiple linear regression); the presence of PAH contributed significantly to the model $(p=0.044)$ while BMI did not. $\beta O H B$ correlated with second phase hepatic extraction at baseline (figure 4c) and throughout the clamp (figure $4 \mathrm{~d}$ ). In agreement with known physiology of lipid oxidation and ketogenesis, acetylcarnitine correlated with $\beta \mathrm{OHB}$ (figure $4 \mathrm{e}$ ). 


\begin{tabular}{|c|c|c|c|}
\hline & Control & PAH & p-value \\
\hline Subjects n & 6 (5 female) & 6 (5 female) & \\
\hline Age years & $40 \pm 15$ & $49 \pm 12$ & 0.32 \\
\hline Height $\mathrm{cm}$ & $171 \pm 7$ & $167 \pm 8$ & 0.36 \\
\hline Weight kg & $90 \pm 18$ & $87 \pm 12$ & 0.75 \\
\hline BMI $\mathrm{kg} \cdot \mathrm{m}^{-2}$ & $31 \pm 4$ & $31 \pm 5$ & 0.73 \\
\hline Baseline glucose $\mathrm{mg} \cdot \mathrm{dL}^{-1}$ & $90 \pm 11.6$ & $86 \pm 3.4$ & 0.40 \\
\hline Baseline insulin $\mu \mathrm{U} \cdot \mathrm{mL}^{-1}$ & $16 \pm 7.1$ & $11 \pm 2.9$ & 0.18 \\
\hline Baseline C-peptide $\mathrm{ng} \cdot \mathrm{mL}^{-1}$ & $2.2 \pm 0.5$ & $2.0 \pm 0.6$ & 0.44 \\
\hline Baseline $\beta$ OHВ mM & $0.16 \pm 0.03$ & $0.22 \pm 0.03$ & 0.02 \\
\hline Baseline FFA $\mu \mathrm{M}$ & $857 \pm 245$ & $961 \pm 408$ & 0.61 \\
\hline HOMA-IR & $3.7 \pm 2.0$ & $2.4 \pm 0.5$ & 0.17 \\
\hline BMR kcals-day ${ }^{-1}$ & $1484 \pm 317$ & $1519 \pm 88$ & 0.82 \\
\hline Baseline RER & $0.80 \pm 0.03$ & $0.79 \pm 0.03$ & 0.81 \\
\hline SBP mmHg & $130 \pm 4$ & $120 \pm 8$ & 0.28 \\
\hline DBP $\mathrm{mmHg}$ & $76 \pm 8$ & $69 \pm 10$ & 0.25 \\
\hline Heart rate beats. $\min ^{-1}$ & $69 \pm 10$ & $81 \pm 13$ & 0.11 \\
\hline $\mathrm{O}_{2}$ saturation $\%$ & $99.2 \pm 1.3$ & $94.5 \pm 2.1$ & $<0.01$ \\
\hline \multicolumn{4}{|l|}{ Liver panel } \\
\hline Total protein $\mathrm{g} \cdot \mathrm{dL}^{-1}$ & $6.8 \pm 0.5$ & $6.6 \pm 0.5$ & 0.65 \\
\hline Albumin $\mathrm{g} \cdot \mathrm{dL}^{-1}$ & $3.9 \pm 0.3$ & $4.0 \pm 0.3$ & 0.84 \\
\hline Total bilirubin $\mathrm{mg} \cdot \mathrm{dL}^{-1}$ & $0.45 \pm 0.14$ & $0.52 \pm 21$ & 0.54 \\
\hline ALP U. $L^{-1}$ & $55 \pm 14$ & $72 \pm 20$ & 0.12 \\
\hline AST $U \cdot L^{-1}$ & $19 \pm 8$ & $14 \pm 4$ & 0.20 \\
\hline ALT U. $L^{-1}$ & $24 \pm 13$ & $14 \pm 7$ & 0.15 \\
\hline
\end{tabular}

Data are presented as mean \pm SD, unless otherwise stated. PAH: pulmonary arterial hypertension; BMI: body mass index; $\beta O H B$ : $\beta$-hydroxybutyrate; FFA: free fatty acids; HOMA-IR: homeostatic model assessment of insulin resistance; BMR: basal metabolic rate; RER: respiratory exchange ratio; SBP: systolic blood pressure; DBP: diastolic blood pressure; $\mathrm{O}_{2}$ saturation: oxygen saturated haemoglobin relative to total haemoglobin; ALP: alkaline phosphatase; AST: aspartate transaminase; ALT: alanine transaminase.

Palmitate (C16:0) correlated strongly with total FFAs $\left(\mathrm{r}^{2}=0.943, \mathrm{p}<0.001\right.$; figure S2a), suggesting palmitate increases in proportion with total FFA concentrations. In contrast, palmitoleate (C16:1) did not correlate with total FFAs $\left(\mathrm{r}^{2}=0.051, \mathrm{p}=0.481\right.$; figure $\left.\mathrm{S} 2 \mathrm{~b}\right)$, suggesting alternate regulation of palmitoleate outside of global FFA concentrations. Further, palmitoleate was strikingly well-correlated with $\beta \mathrm{OHB}$ (figure $4 \mathrm{f}$ ) and $\mathrm{M} / \mathrm{I}$ (figure S1c and d), implicating a potential relationship with nutrient metabolism in agreement with emerging literature on palmitoleate as a bio-active lipid [17].

\section{Correlational analysis with clinical measures}

Table 2 details clinical characteristics of PAH participants. Given the unexpected metabolic findings and the prevailing hypothesis that aberrant metabolism is inherent to PAH pathophysiology, we assessed whether the metabolic abnormalities were associated with functional capacity (6-min walk distance) or measures of right heart dysfunction. 6-min walk distance correlated with both the first (figure S3a) and second phase (figure S3b) hepatic extraction, and with FFA: $\beta O H B$ (figure S3c). Presence of pericardial effusion correlated with greater $\beta \mathrm{OHB}$ during the clamp (figure $\mathrm{S} 3 \mathrm{~d}$ ). Presence of right atrial dilation correlated with lower hepatic extraction (figure S3e). FFAs during the clamp were associated with higher resting heart rate (figure S3f), while baseline FFAs were related to lower $\mathrm{O}_{2}$ saturation (figure S3g). M/I correlated with both right ventricular systolic pressure (figure S3h) and $\mathrm{N}$-terminal B-type natriuretic peptide (NT-proBNP) (figure S3i).

\section{Plasma metabolomics}

To validate the clamp metabolic findings and clinical associations in a larger cohort, untargeted metabolomic analysis was conducted on 21 idiopathic PAH patients and 31 age-, BMI- and sex-matched controls. Subject characteristics of the metabolomics cohort and scaled intensity values of all 862 targets are available elsewhere [11]. We identified 862 metabolites, of which 358 were different in PAH (213 elevated, 145 reduced). A volcano plot illustrating the metabolite responses are displayed in figure 5a. A heat map displaying the top 30 differentially expressed metabolites is illustrated in figure 5b. Notably, $\beta O H B$ was five-fold higher in $\mathrm{PAH}(\mathrm{q}=0.001$, figure $5 \mathrm{c}) .30$ metabolites represent fatty acid oxidation 

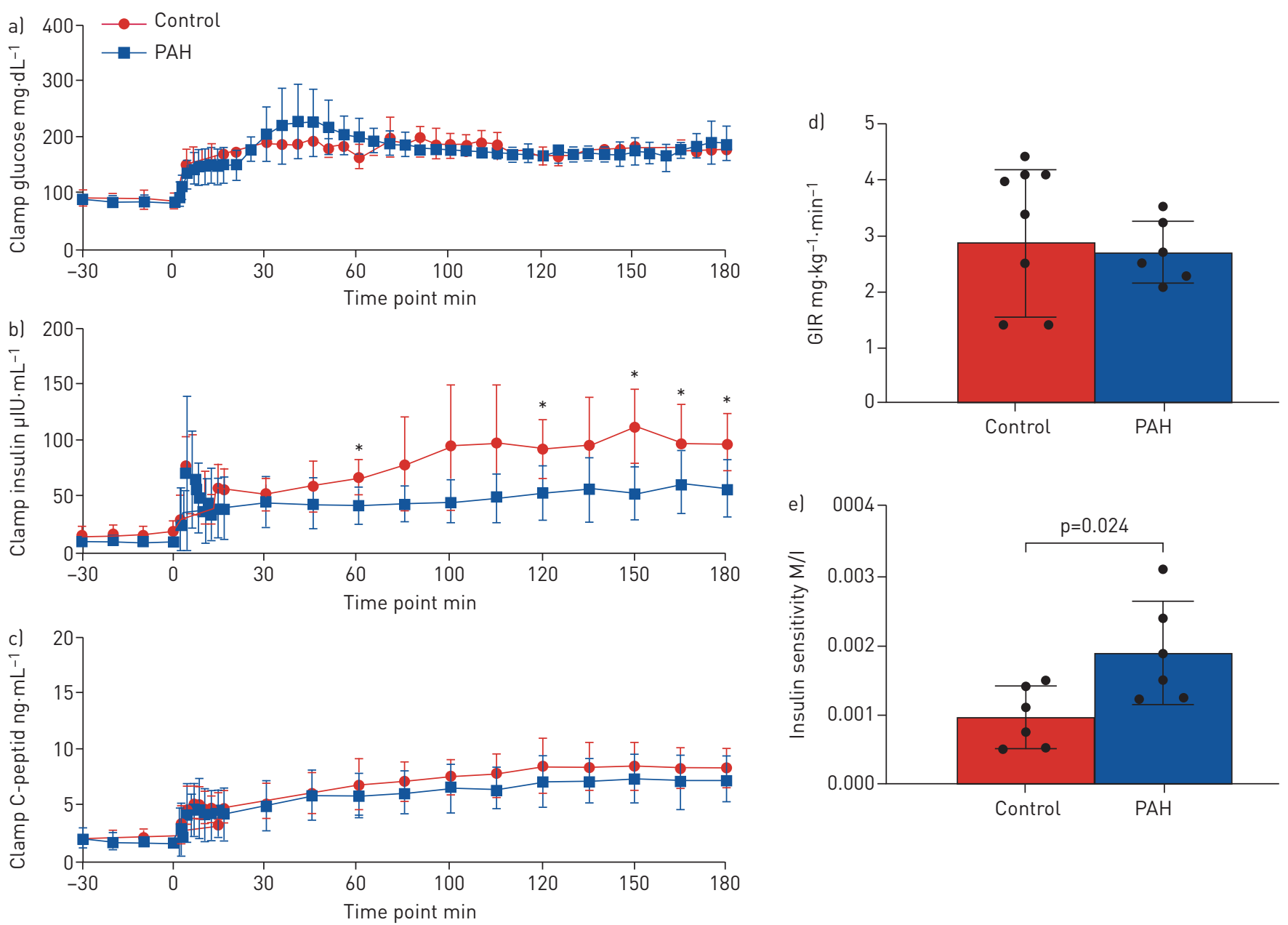

FIGURE 1 Hyperglycaemic clamp. Hyperglycaemic clamp results for glucose (a), insulin (b) and C-peptide (c). Research participants were effectively clamped at $180 \mathrm{mg} \cdot \mathrm{dL}^{-1}$ glucose. a) 150-180 min, pulmonary arterial hypertension (PAH): $177 \pm 4 \mathrm{mg} \cdot \mathrm{dL}^{-1}$, control: $179 \pm 1 \mathrm{mg} \cdot \mathrm{dL}^{-1}$; $\mathrm{ns}$. Insulin concentrations were reduced in PAH compared with control. b) $150-180 \mathrm{~min}$, PAH: $55.2 \pm 10.3 \mu \mathrm{IU} \cdot \mathrm{mL}^{-1}$, control: $102.8 \pm 12.2 \mu \mathrm{IU} \cdot \mathrm{mL}^{-1}$; two-way repeated measures ANOVA (groupxtime), significant effect of time and interaction. C-peptide concentrations were similar between groups. c) 150-180 min, PAH: $7.4 \pm 0.9 \mathrm{ng} \cdot \mathrm{mL}^{-1}$, control: $8.5 \pm 0.8 \mathrm{ng} \cdot \mathrm{mL}^{-1}$; two-way repeated measures ANOVA (groupxtime), significant effect of time. *: Tukey's post hoc test $\mathrm{p}<0.05$. d) Glucose infusion rate was similar between groups $\left(\mathrm{PAH}: 2.7 \pm 0.2 \mathrm{mg} \cdot \mathrm{kg}^{-1} \cdot \mathrm{min}^{-1}, \mathrm{control}: 2.9 \pm\right.$ $0.5 \mathrm{mg} \cdot \mathrm{kg}^{-1} \cdot \mathrm{min}^{-1}$ ). e) Insulin sensitivity (M/I) was elevated in PAH compared with controls (M/I, 150-180 min; PAH: 0.025 \pm 0.007, control: $0.013 \pm$ $0.008, \mathrm{p}=0.024$. $\beta$ OHB: $\beta$-hydroxybutyrate; $\mathrm{M} / \mathrm{l}, \mathrm{mg}$ glucose $/ \mathrm{kg}$ body weight $\cdot \mathrm{min}^{-1} \cdot \mu \mathrm{U}$ insulin $\cdot \mathrm{mL}^{-1}$.

(acylcarnitines); 21 were elevated in $\mathrm{PAH}$, including acetylcarnitine (1.7-fold, $\mathrm{q}<0.001$; figure 5d). Mirroring clamp data, $\beta \mathrm{OHB}$ and acetylcarnitine strongly correlated (figure S4a). Of 38 fatty acid metabolites, 19 were elevated in $\mathrm{PAH}$, including palmitate (figure $\mathrm{S} 4 \mathrm{~b}$ ), while palmitoleate showed the greatest increase (figure S4c). Seven amino acids have ketogenic capacity of which six were reduced in PAH (trp, tyr, ile, thr, lys, leu; all $\mathrm{q}<0.10$; figure S4d). Correlation analysis was conducted between metabolites of interest identified from the clamp study and clinical measures. Right ventricular systolic pressure was associated with $\beta \mathrm{OHB}$ (figure S5a), palmitate (figure S5b) and acetylcarnitine (figure S5c). Grade of tricuspid regurgitation correlated with $\beta \mathrm{OHB}$ (figure S5d) and palmitate (figure S5e). Palmitate was negatively associated with myocardial oxygen consumption (figure S5f). Total free fatty acids were not available for this metabolomics dataset, thus palmitate was used as a surrogate comparison due to its known strong correlation to total FFAs [18] and independent validation in the clamp cohort in this report (figure S2a).

\section{Discussion}

This is the first work to use the hyperglycaemic clamp to investigate $\beta$-cell function in individuals with idiopathic PAH. We confirmed a decreased insulin response to hyperglycaemia in PAH, corroborating our findings from an oral glucose tolerance test [9]. The novel contribution of this work is that the reduced insulin response to hyperglycaemia was not attributable to reduced pancreatic $\beta$-cell insulin secretion or skeletal muscle insulin sensitivity compared with age-, BMI- and sex-matched controls. Rather, hepatic insulin extraction was significantly elevated in $\mathrm{PAH}$, suggesting hepatic insulin extraction may contribute 


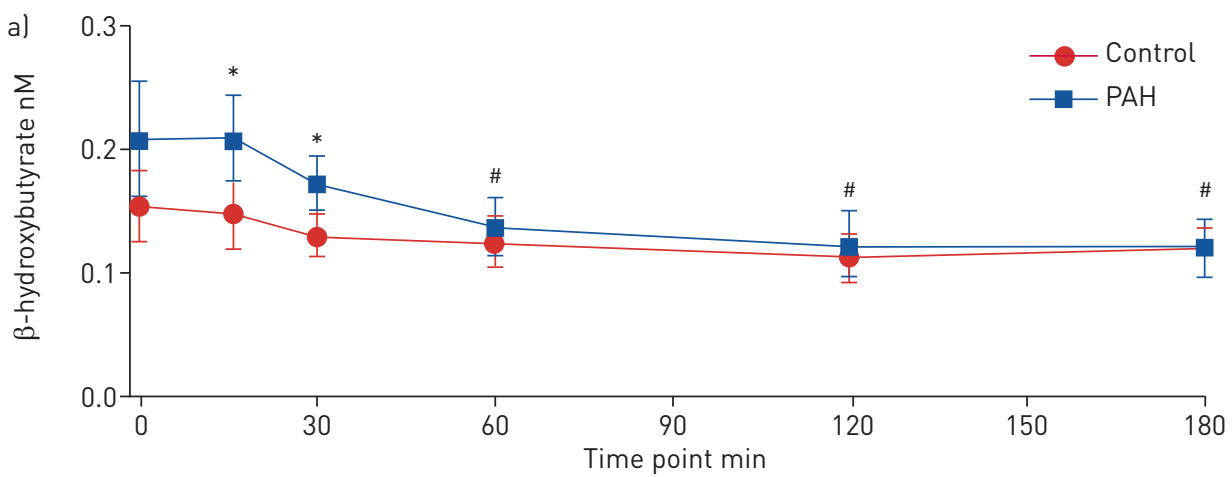

b)

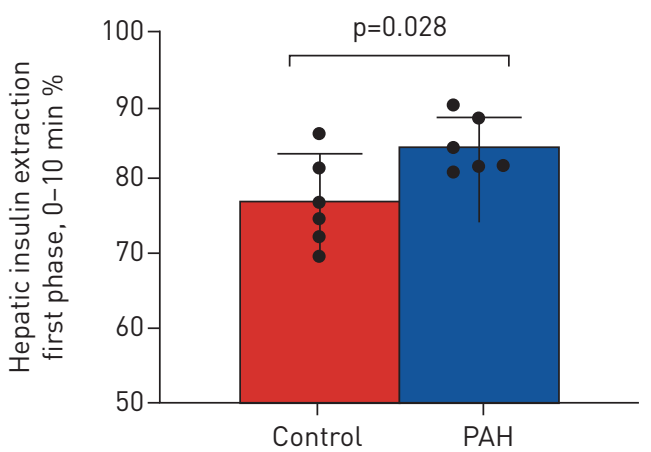

c)

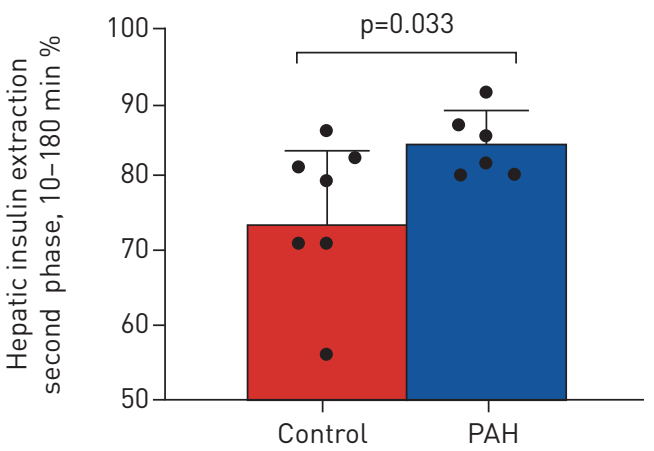

FIGURE $2 \beta$-Hydroxybutyrate and hepatic insulin extraction. a) The ketone $\beta$-hydroxybutyrate was elevated in pulmonary arterial hypertension (PAH) through $30 \mathrm{~min}$ of hyperglycaemia, reaching similar levels as controls by $60 \mathrm{~min}$. (PAH: baseline $0.22 \pm 0.02 \mathrm{mM}, 180 \mathrm{~min} 0.12 \pm 0.01 \mathrm{mM}$; control: baseline $0.16 \pm 0.01 \mathrm{mM}, 180 \mathrm{~min}$ $0.12 \pm 0.01 \mathrm{mM}$; two-way repeated measures ANOVA, group $\mathrm{p}=0.018$, time $p<0.001$, interaction $p<0.001$ ). *: different between groups, Tukey's post hoc test $p<0.05$; : different from baseline within PAH group, Dunnett's multiple comparisons test $p<0.02$. Hepatic insulin extraction was significantly greater in $P A H$ compared with controls during the first phase insulin response (b) $0-10 \mathrm{~min}$; $\mathrm{PAH}: 84.7 \pm 1.5 \%$, control: 77.1 $\pm 2.5 \%, p=0.028$ and through prolonged hyperglycaemia (c) $10-180 \mathrm{~min} ; \mathrm{PAH}: 84.4 \pm 1.8 \%$, control: $73.5 \pm 2.5 \%$, $\mathrm{p}=0.033$; significance determined by unpaired Student's t-test. Hepatic insulin extraction, molar ratio of plasma insulin/plasma C-peptide expressed as a percentage.

to the poor oral glucose tolerance in observed PAH pathophysiology. Another primary contribution of this work was defining preferential lipid and ketone metabolism associated with PAH clinical measures, along with replicating these findings in a larger cohort using a plasma metabolomics approach. Fatty acid oxidation (acetylcarnitine) and ketogenesis $(\mathrm{BOHB})$ were elevated in $\mathrm{PAH}$, while maintaining a greater propensity to convert available free fatty acids to ketones (defined by the FFA: $\beta$ OHB ratio) was associated with improved exercise endurance, an important clinical marker in PAH. These data suggest an adaptive shift in nutrient metabolism towards fatty acid and ketone utilisation at the expense of glucose control in PAH.

Our interpretations are based upon the framework that glucose control is primarily regulated by the hormone insulin, governed by three critical control points: pancreatic insulin secretion; hepatic insulin extraction; and skeletal muscle insulin sensitivity. Upon induction of hyperglycaemia, insulin is secreted by the pancreatic $\beta$-cells. Prior to reaching arterial circulation, insulin is partially sequestered by hepatic insulin extraction, modifying the circulating insulin concentration. Finally, circulating insulin acts on peripheral tissues to facilitate tissue-specific glucose disposal, skeletal muscle being the largest consumer, whereby the insulin sensitivity of skeletal muscle partially dictates the magnitude of this response. By this manner, these three primary tissues are factors underlying glucose control in PAH: pancreatic insulin secretion, hepatic insulin extraction and skeletal muscle insulin sensitivity. An integrated working model of our findings at these critical control points is illustrated in figure 6, including equivalent pancreatic insulin secretion, elevated hepatic insulin extraction and increased skeletal muscle insulin sensitivity in $\mathrm{PAH}$ compared with age-, BMI- and sex-matched controls.

\section{Liver: insulin extraction and ketogenesis}

Important to the interpretation of these results, the control group was overweight/obese and sedentary and presented with classic signs of early stages of insulin resistance: elevations in FFAs and insulin secretion 

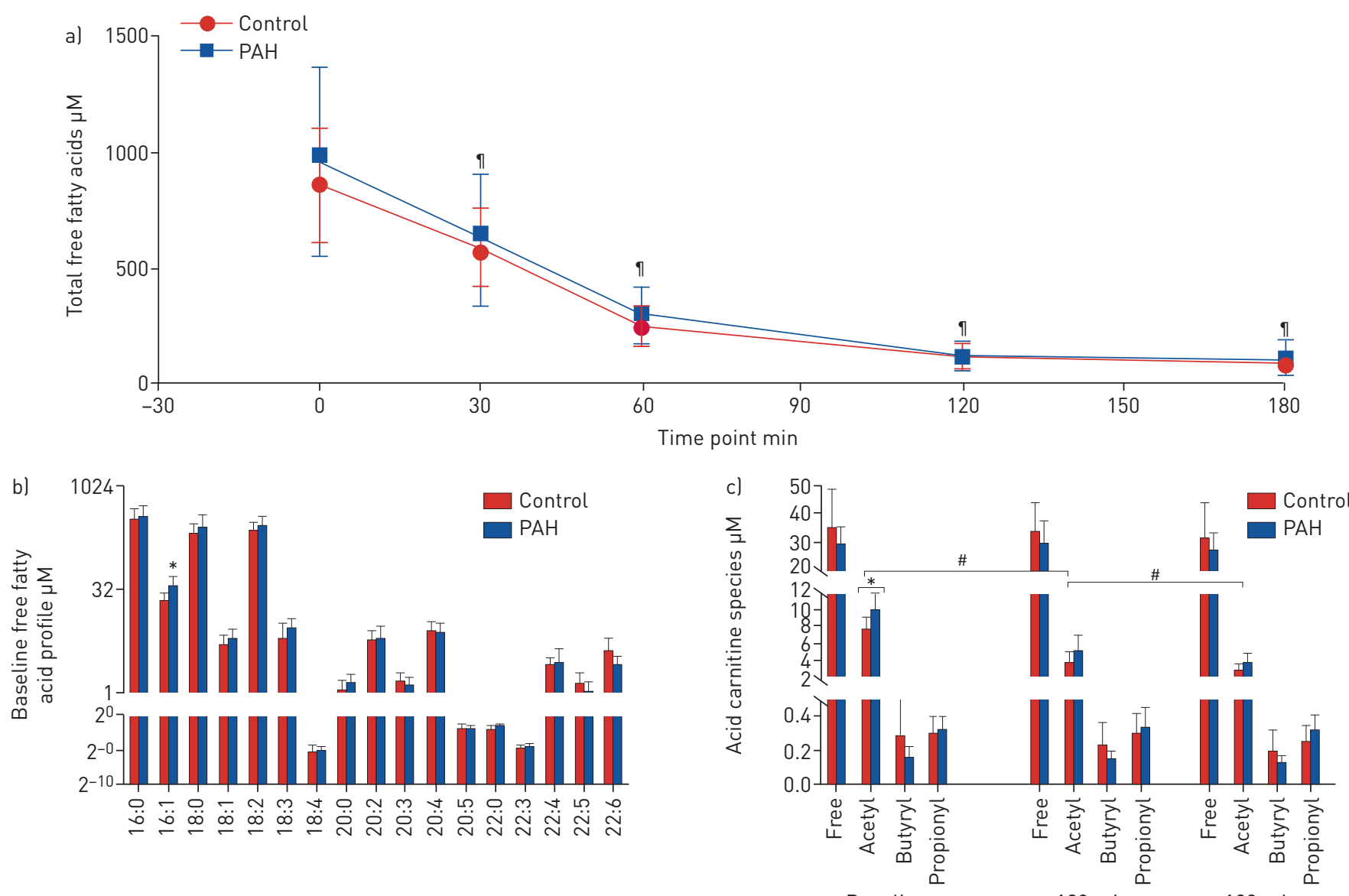

Baseline

$120 \mathrm{~min}$

$180 \mathrm{~min}$

FIGURE 3 Free fatty acids and lipid oxidation. a) Total plasma free fatty acids were similar between groups at baseline (pulmonary arterial hypertension (PAH): $961 \pm 167 \mu \mathrm{M}$; control: $857 \pm 100 \mu \mathrm{M}$ ) and decreased equally with hyperglycaemia (180 min; PAH: $111 \pm 30 \mu \mathrm{M}$, control: $89 \pm 13 \mu \mathrm{M}$; two-way repeated measures ANOVA, time $\mathrm{p}<0.001)$. b) Lipidomic profiling of free fatty acids revealed elevated palmitoleate (16:1) in PAH (PAH: $37.3 \pm 4.9 \mu \mathrm{M}$; control: $22.1 \pm 2.9 \mu \mathrm{M} ; \mathrm{p}=0.023$ ). c) Free-, butyryl- and propionyl-carntines were similar between groups and remained unchanged at 120 and $180 \mathrm{~min}$ of hyperglycaemia. Acetylcarnitine was elevated in $\mathrm{PAH}$. Both groups decreased acetylcarnitine similarly in response to hyperglycaemia. (PAH: baseline $10.2 \pm 0.7 \mu \mathrm{M}, 120 \mathrm{~min} 5.3 \pm 0.6 \mu \mathrm{M}, 180 \mathrm{~min} 3.9 \pm 0.4 \mu \mathrm{M}$; control baseline $7.7 \pm 0.5 \mu \mathrm{M}, 120 \mathrm{~min} 4.0 \pm 0.4 \mu \mathrm{M}, 180 \mathrm{~min}$ $3.0 \pm 0.2 \mu \mathrm{M}$, two-way repeated measures ANOVA, group $p=0.033$, time $p<0.001$, interaction $p<0.001$, Tukey's multiple comparisons tests, within group (Baseline versus 120 min versus $180 \mathrm{~min}$ ), all $p<0.05$ ). *: different between groups $p<0.05$; "\#: different from baseline $p<0.05$; ${ }^{\text {१: }}$ Sidak's multiple comparison test, different from baseline, $p<0.05$.

with reductions in fasting $\beta \mathrm{OHB}[19,20]$ and hepatic insulin extraction $[21,22]$. Pancreatic $\beta$-cell function (i.e. insulin secretion) was similar between groups, suggesting $\mathrm{PAH}$ patients are progressing along the natural history of diabetes. Typically, hepatic extraction is progressively reduced along this continuum, thus, the finding of greater hepatic insulin extraction in PAH was unexpected. To our knowledge, this is the first report suggesting PAH presents with elevated hepatic insulin extraction in response to hyperglycaemia. Given neither pancreatic $\beta$-cell insulin secretion nor skeletal muscle insulin sensitivity were indicative of poor glucose control, it remains plausible that the elevation in hepatic insulin extraction plays a role in the poor oral glucose control observed in PAH [23]. Future research should utilise more direct approaches to assess hepatic insulin extraction, such as stable isotopic tracers, invasive portal vein sampling or rigorous modelling calculations in combination with an oral glucose tolerance test [24], to further pursue this line of inquiry in PAH metabolism.

Another important role of the liver in metabolic health is hepatic ketogenesis. Fasted circulating $\beta \mathrm{OHB}$ reflects hepatic ketogenesis [25], thus the elevated $\beta \mathrm{OHB}$ observed in the $\mathrm{PAH}$ group is indicative of increased ketogenesis. This agrees with the literature on the metabolism of heart failure $[12,26]$ where ketones are preferentially utilised by cardiac tissue as an adaptive response to declining heart function [27], as ketones are a more energetically favourable fuel source [28]. Fasting hepatic ketogenesis also positively correlated with hepatic insulin extraction in this population. This relationship between these two hepatic processes is both novel and intriguing because ketogenesis is partly regulated by insulin. Yet, it remains unknown whether the insulin extracted by hepatocytes remains inert prior to degradation, or instead, first induces local insulin signalling within hepatocytes (e.g. inhibiting ketogenesis). 

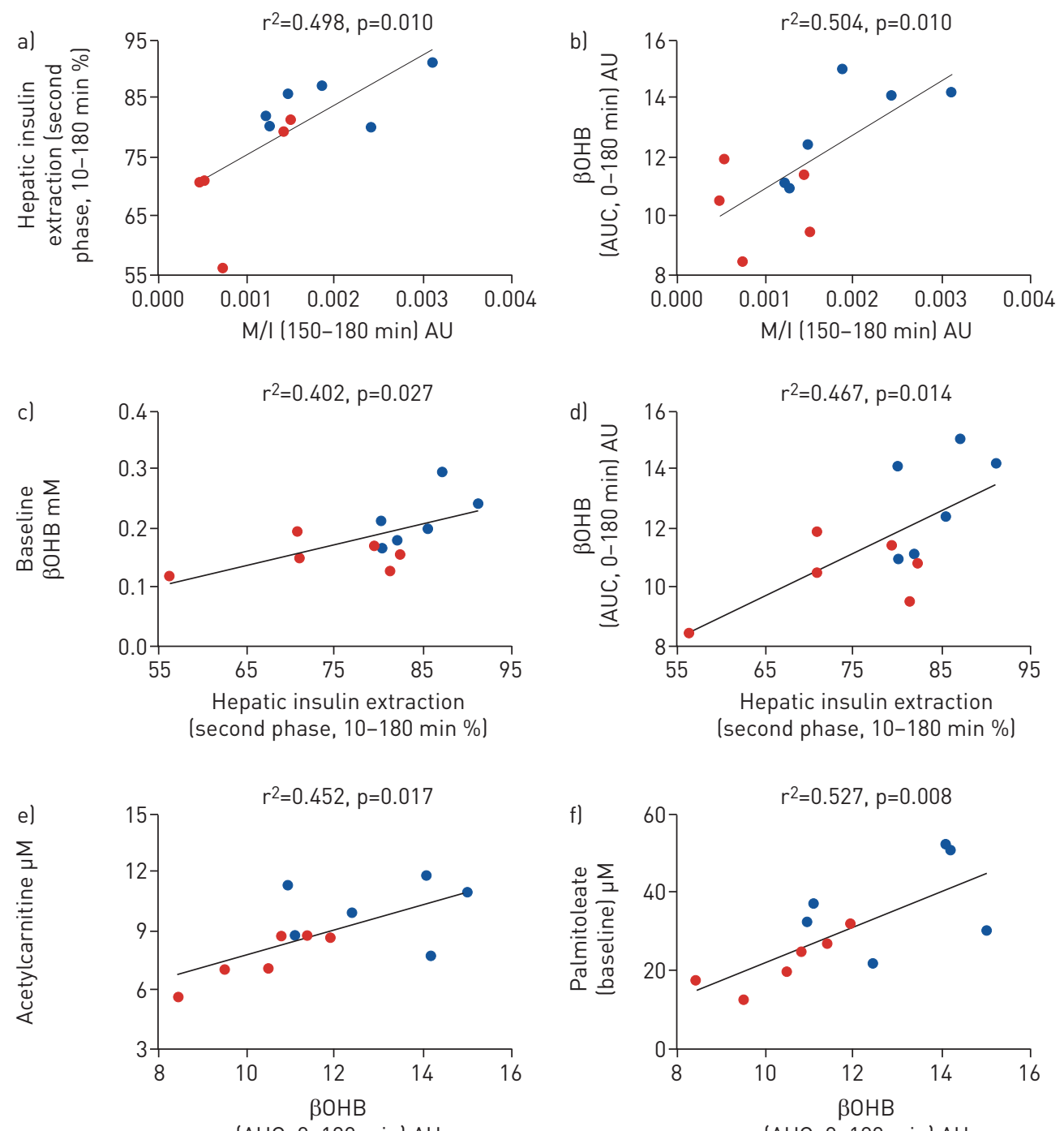

(AUC, 0-180 $\mathrm{min}$ ) AU

(AUC, $0-180 \mathrm{~min}$ ) AU

FIGURE 4 Correlational analysis with metabolic measures. To gain insight into nutrient metabolism in pulmonary arterial hypertension (PAH), we interrogated relationships between the primary metabolic findings. Insulin sensitivity (M/I) correlated strongly with the (a) hepatic insulin extraction (second phase, $r^{2}=0.498$, $p=0.010)$ and (b) $\beta$-hydroxybutyrate $(\beta O H B)\left(r^{2}=0.504, p=0.010\right)$ during the clamp. $\beta O H B$ concentrations correlated with second phase hepatic extraction at (c) baseline $\left(r^{2}=0.402, p=0.027\right)$ and (d) throughout the clamp $\left(r^{2}=0.467, p=0.014\right)$. $\beta O H B$ expectedly correlated with (e) acetylcarnitine $\left(r^{2}=0.452, p=0.017\right)$ and additionally with (f) palmitoleate $\left(r^{2}=0.527, p=0.008\right) . M / I, m g$ glucose $\cdot k g$ body weight ${ }^{-1} \cdot \mathrm{min}^{-1} \cdot \mu U$ insulin $\cdot \mathrm{mL}^{-1}$.

An additional regulator of ketogenesis is delivery of FFAs to the liver via adipose tissue lipolysis [29], whereby increased FFAs are associated with increased ketones in healthy physiology. To address whether the observed elevation in fasting hepatic ketogenesis was merely a reflection of elevated FFAs, we measured fasting free fatty acids, showing no differences between groups. To address whether the ability to convert prevailing free fatty acids to ketones was related to clinical outcomes in $\mathrm{PAH}$, we calculated a ratio of free fatty acids to ketones (FFA: $\beta \mathrm{OHB}$ ) and assessed correlations with primary clinical measures. Independently, FFA or $\beta \mathrm{OHB}$ are associated with poorer clinical measures in this study. In contrast, the FFA: $\beta \mathrm{OHB}$ ratio was negatively associated with clinical severity, suggesting that a greater ability to convert available FFAs to ketones is indicative of better clinical health in PAH. Although ketones have previously been viewed as a mere by-product of metabolism, recent research has implicated ketones as a regulating factor in glucose and lipid metabolism [30], along with possessing independent signalling properties [31]. Taken together, the FFA: $\beta \mathrm{OHB}$ ratio may provide a novel characterisation of metabolism in both $\mathrm{PAH}$ and isolated metabolic disease. Further, the physiological relationship between $\beta \mathrm{OHB}$ concentrations and hepatic insulin extraction have, to our knowledge, not been previously reported. This provides incentive to investigate the relationship between hepatic ketogenesis and hepatic insulin extraction in other clinical settings. 
TABLE 2 Clinical characteristics of PAH patients (clamp study, $n=6$ )

Measured value

\begin{tabular}{lc} 
NYHA class & \\
II & \\
III & 1 \\
Echocardiography & 5 \\
RV dilation present & 2 \\
RV dysfunction present & 3 \\
RA dilation present & $71.3 \pm 12.8$ \\
RVSP mmHg & 3 \\
Pericardial effusion present & $80.8 \pm 24.4$ \\
NT-proBNP pg-mL ${ }^{-1}$ & \\
Medications & 1 \\
Sildenafil & 3 \\
Taldalafil & 1 \\
Bosentan & 2 \\
Macitentan & 5 \\
Treprostinil & $411.7 \pm 25.6$ \\
6MWT m & \\
Right heart catheter data & $8.2 \pm 1.3$ \\
RAP mmHg & $48.2 \pm 4.8$ \\
mPAP mmHg & $11.5 \pm 2.4$ \\
PAWP mmHg & $3.08 \pm 0.18$ \\
Cardiac index L-min ${ }^{-1} \cdot m^{2}$ & $6.2 \pm 0.7$ \\
PVR Wood units & \\
\hline Data are presented as $n$ & \\
Association functional class; RV: right ventricle; RA: right atrium; RVSP: right ventricular systolic pressure; \\
NT-proBNP: N-terminal pro B-type natriuretic peptide; 6MWT: 6-minute walk test; RAP: right atrial \\
pressure; mPAP: mean pulmonary arterial pressure; PAWP: pulmonary arterial wedge pressure; PVR: \\
pulmonary vascular resistance. & \\
\hline
\end{tabular}

\section{Skeletal muscle: insulin sensitivity}

Glucose infused during the clamp is actively transported into skeletal muscle, and in the case of the hyperglycaemic clamp, provides a proxy estimate of skeletal muscle insulin sensitivity (M/I) [15]. This measure of insulin sensitivity is classically reduced in concert with oral glucose tolerance, where individuals with poor oral glucose tolerance are expected to also have poor insulin sensitivity. We and others have previously identified poor oral glucose tolerance in $\mathrm{PAH}[9,13]$, thus it was an unexpected to observe increased skeletal muscle insulin sensitivity in PAH compared with controls. Several explanations for elevated insulin sensitivity in PAH may exist. First, pulmonary arterial hypertension increases basal metabolic rate [9], which was observed in this study when controlling for height, weight, age and sex. We therefore performed an additional analysis to control insulin sensitivity for basal metabolic rate, with unremarkable effects (figure S6a). Another explanation may be an effect of hypoxia in PAH or the overweight/obese control group, which may enhance [32] or impair [33] insulin sensitivity. It is also possible that medications specific to PAH increase insulin sensitivity or blood flow, however, we did not find any relationships with medications and insulin sensitivity within this small cohort.

The FFA: $\beta O H B$ ratio was associated with improved clinical measures and predicted $\mathrm{M} / \mathrm{I}$ after controlling for BMI and PAH status. This suggests the hepatic capacity to convert FFAs to ketones may be a novel factor in skeletal muscle insulin sensitivity. Insulin sensitivity was also predicted by hepatic extraction, which is in agreement with recent human literature [34] and instils additional confidence in the validity of these unexpected results. Reductions in ketogenic amino acids in concert with elevated ketones is particularly noteworthy, as fasted-state amino acids primarily originate from muscle tissue and loss of muscle mass is common in advanced PAH and heart failure. This observation begs the question as to whether exogenous provision of ketogenic precursors could protect $\mathrm{PAH}$-induced muscle catabolism and should be pursued further.

Ultimately, larger studies utilising clamp-derived insulin sensitivity are warranted to verify the observation of elevated skeletal muscle insulin sensitivity in PAH compared to age, BMI and sex-matched controls. The addition of a lean, healthy control group, representative of normal skeletal muscle insulin sensitivity, would substantially strengthen the study design. Importantly, identifying the metabolism underlying poor 

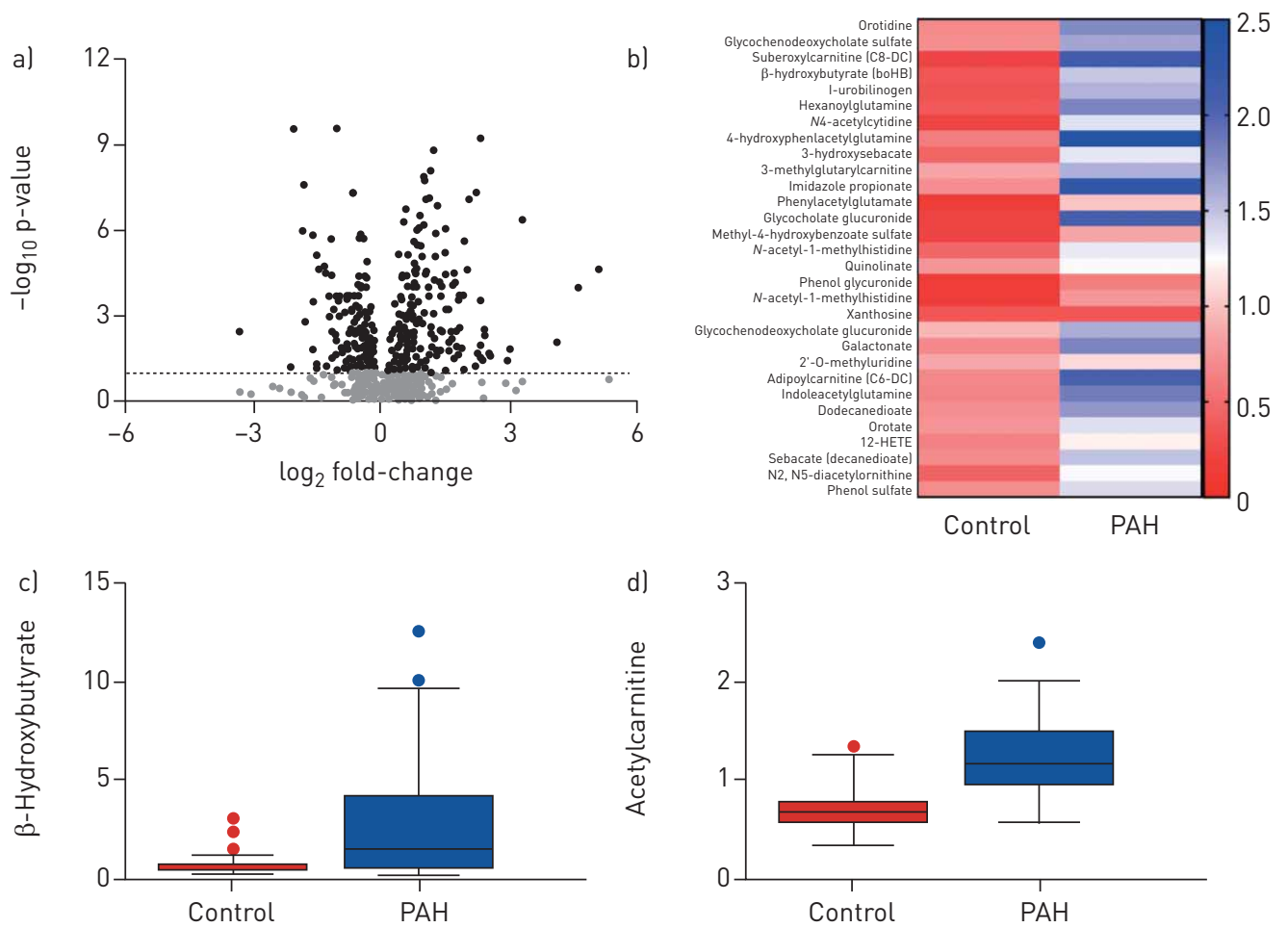

FIGURE 5 Untargeted plasma metabolomics. a) Volcano plot lexcluding metabolites from drug or environmental exposure) representing fold-change of log scaled data, $q<0.10$. b) Heat map of top 30 differentially expressed metabolites. Both (c) $\beta$-hydroxybutyrate $(\beta \mathrm{OHB})$ and (d) acetylcarnitine were elevated in pulmonary arterial hypertension (PAH) compared with control. Box-and-whiskers plots presented using the Tukey method.

oral glucose tolerance in the context of increased skeletal muscle insulin sensitivity would be an impactful contribution to our understanding of PAH pathophysiology.

\section{FFAs and fat oxidation}

Fasting FFAs were similar between groups, but notably, higher than levels typically reported for healthy, non-obese individuals $[35,36]$. PAH and controls reduced FFAs similarly during the clamp and, given FFA concentrations are primarily driven by rates of adipose tissue lipolysis, this suggests the adipose tissue of PAH and controls respond similarly to insulin. To gain deeper insight into fatty acid metabolism, we performed short-chain acylcarnitine analysis. Acetylcarnitine, an indicator of complete fatty acid oxidation, was elevated in $\mathrm{PAH}$ and was associated with $\beta \mathrm{OHB}$ in both clamp and metabolomics cohorts, supporting the interpretation of preferential lipid and ketone metabolism in PAH. An interesting finding was elevated palmitoleate in $\mathrm{PAH}$, which has been reported to impart beneficial effects on insulin sensitivity and cardiovascular health [37], providing a potential mechanism for the elevated insulin sensitivity in PAH and adding to the growing interest of palmitoleate in metabolic research.

\section{Clinical correlates and implications}

Distance walked during a 6-min walk test is an important predictor of mortality and clinical outcomes in $\mathrm{PAH}$, and in the current study was correlated with hepatic insulin extraction, implicating a role for nutrient metabolism in PAH clinical outcomes, not just metabolic health. In both clamp and metabolomic cohorts, FFAs and $\beta \mathrm{OHB}$ were independently associated with poorer clinical measures, while the FFA: $\beta \mathrm{OHB}$ ratio correlated with improved clinical measures. In the context of the literature, these results suggest increased FFAs and $\beta \mathrm{OHB}$ are an early metabolic adaptation in response to the heart failure associated with $\mathrm{PAH}$.

A prioritisation of FFA and $\beta \mathrm{OHB}$ fuel sources requires circulating insulin to be maintained at lower concentrations. In physiological agreement, we observed markedly increased skeletal muscle insulin sensitivity in PAH, permitting equivalent glucose disposal with less than half the insulin concentrations of controls. The factors responsible for maintaining reduced insulin in PAH physiology are unclear, though analysis of C-peptide and insulin relationships implicates elevated hepatic insulin extraction. The 


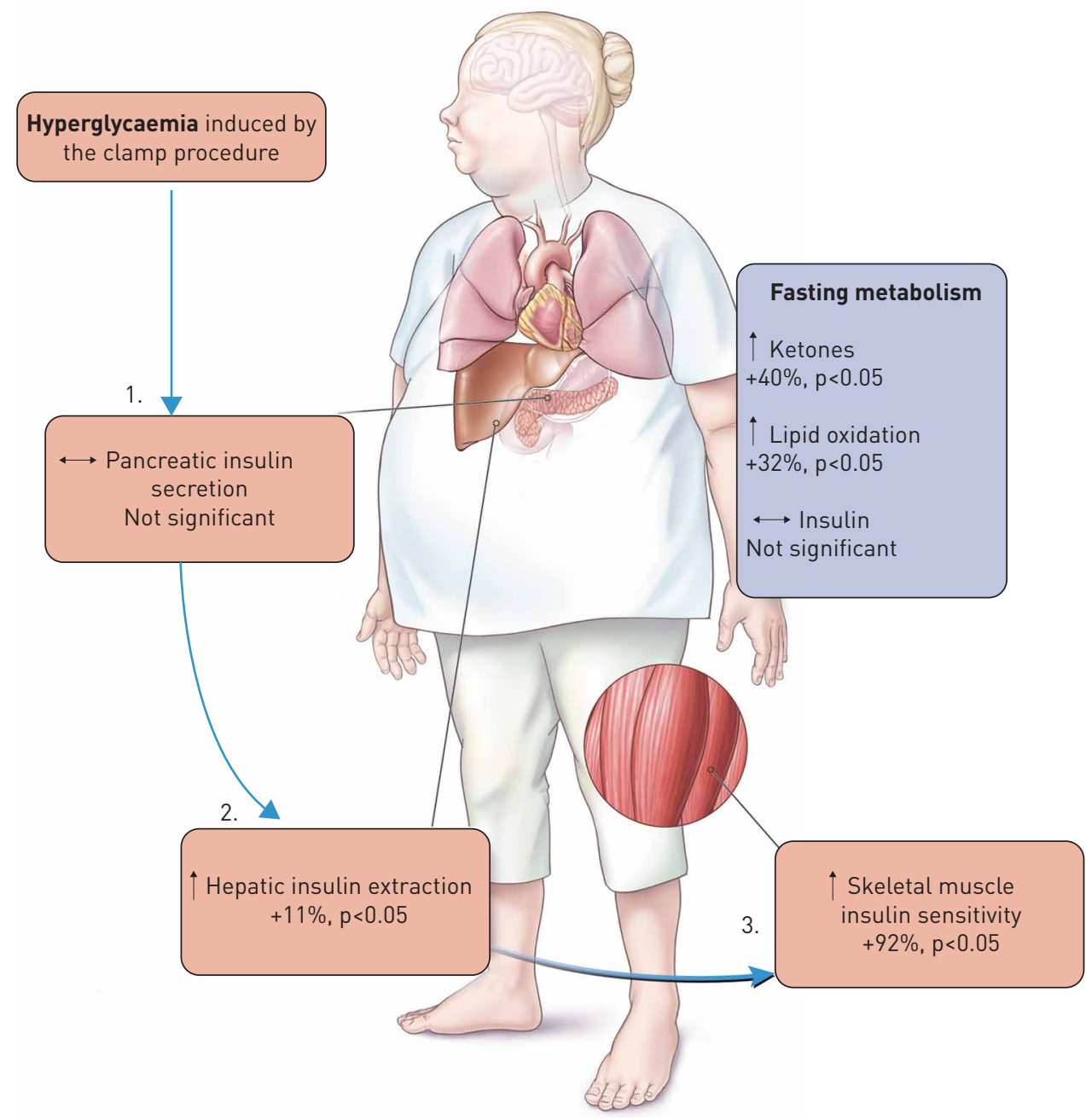

FIGURE 6 Summary of metabolic findings in pulmonary arterial hypertension (results compared with age-, sex- and body mass index-matched controls). The underlying physiology of pulmonary arterial hypertension (PAH) is characterised by elevated blood pressure in the arteries of the lungs, which puts stress on the right ventricle, culminating in progressive heart failure. Poor oral glucose control in PAH has been observed in the literature, but the underlying factors remain to be fully elucidated. Importantly, glucose control is primarily regulated by the hormone insulin, which dictates the disposal of glucose from the blood into peripheral tissues. The impact of insulin-mediated glucose control in response to hyperglycaemia is primarily regulated by three critical control points: pancreatic $\beta$-cell insulin secretion, hepatic insulin extraction and skeletal muscle insulin sensitivity. Here, we assessed fasting metabolism in PAH along with the insulin response to hyperglycaemia induced by the gold-standard hyperglycaemic clamp technique. In PAH, fasting metabolism was shifted towards utilisation of ketones and lipids, despite similar insulin levels compared to controls. During experimentally induced hyperglycaemia, we observed reduced circulating insulin in PAH (fig. 1b). We further observed that 1) pancreatic $\beta$-cell insulin secretion was equivalent between PAH and controls, 2) hepatic insulin extraction was elevated in PAH and 3) skeletal muscle insulin sensitivity was increased in PAH.

regulation of hepatic extraction is not well understood $[38,39]$, but several possibilities exist, including liver congestion [40] caused by elevated right atrial pressure, autonomic control of the liver [41], changes in liver blood flow [29] or side effects of PAH medications [42].

It also remains to be resolved if these metabolic observations are regulated in coordinated fashion or merely coincidental findings related to the complex physiology and pharmacotherapy in PAH. However, we speculate that elevated hepatic insulin extraction may be a physiologic means to maintain low insulin levels in the context of elevated peripheral insulin sensitivity and thus support ketone metabolism to supply fuel-efficient ketones to cardiac tissue [27, 28], an adaptation that may be necessary to compensate for declining heart function, but which inadvertently contributes to poor glucose control in individuals with PAH and overweight/obesity. 
These data agree with the left heart failure literature, evidencing sustained $\beta$-cell function with elevated hepatic insulin extraction [43]. However, the left heart failure literature primarily uses oral glucose testing and typically shows lower fasting insulin levels in populations with more severe heart failure compared with our study population. The novelty in this study is the use of the hyperglycaemic clamp, which by design, creates hyperglycaemia while avoiding the contribution of gut factors (i.e. gut peptides) and gastric emptying present with oral glucose procedures. The observed reductions in circulating insulin and elevated hepatic insulin extraction in response to hyperglycaemia support the repurposing of some anti-diabetic agents for consideration in future $\mathrm{PAH}$ research. Special interest should be noted for the sodium glucose co-transporter-2 (SGLT2) inhibitors or glucagon-like peptide 1 (GLP1) receptor agonists, as they improve glucose control while having independent cardioprotective effects [44]. Similarly, research is needed to determine the impact of medical nutrition therapy to reduce glucose excursions and support lipid and ketone metabolism on PAH-related outcomes.

\section{Limitations}

Due to complexity and patient burden, the hyperglycaemic clamp was limited to a sample size of 12 participants, yet we show strong reproducibility of results with prior work. Furthermore, we validated the primary findings in a separate cohort of 52 participants using unbiased plasma metabolomics. Our interpretations on nutrient metabolism were based on plasma concentrations and thus, we cannot address nutrient flux (production versus disposal) or other potentially confounding factors like endogenous glucose production, glucose effectiveness or lipid turnover, which require stable isotope tracer approaches. Hepatic/ extrahepatic extraction is difficult to directly quantify without portal vein sampling, thus we utilised a commonly reported surrogate measure (molar ratio of insulin:C-peptide) [45, 46]; additional considerations and limitations on this calculation of hepatic insulin extraction are available elsewhere [47, 48]. M/I assumes a linear response of glucose disposal to increasing insulin concentrations, which has been evidenced within the insulin concentrations observed in this study and remains commonly reported from hyperglycaemic clamps $[49,50]$. Hyperinsulinaemic-euglycaemic clamps in combination with oral glucose tolerance testing should be used to verify these results. The clamp cohort was primarily female, and although this is consistent with the predominantly female population of individuals with $\mathrm{PAH}$, we conducted a separate analysis removing the male participants. This analysis revealed unremarkable effects (table S1). Finally, elevated basal metabolic rate is common to $\mathrm{PAH}$ pathology and may impact nutrient metabolism, including insulin sensitivity and ketogenesis. We therefore performed an additional analysis to adjust $\mathrm{M} / \mathrm{I}$ and $\beta \mathrm{OHB}$ for basal metabolic rate, again showing unremarkable effects (figure S6ab). Despite these limitations, this research provides novel insight into the underlying metabolism present in PAH pathophysiology.

\section{Conclusion}

This research provides finer detail of the metabolic underpinnings of PAH, and suggests that the poor oral glucose control is impacted by elevated hepatic insulin extraction rather than defects in pancreatic $\beta$-cell function or skeletal muscle insulin sensitivity. This is the first work to utilise the highly technical hyperglycaemic clamp to assess $\beta$-cell function in $\mathrm{PAH}$, isolating the insulin response to hyperglycaemia per se. Finally, these data support the narrative that the shift in nutrient metabolism (preferential lipid and ketone utilisation at the expense of glucose control) is a beneficial adaptation to compensate for the failing right heart. The metabolic implications of this work may help inform future trials investigating therapeutic approaches to improve PAH outcomes, including supporting lipid and ketone metabolism and minimising glucose excursions through dietary, lifestyle or pharmacological strategies.

Acknowledgements: We thank all our research participants, our funding sources, the Clinical Research Unit staff for assistance with subject screening, clinical visits and hyperglycaemic clamp support and Charles Hoppel and Henri Brunengraber for expert technical advice and analysis.

Author contributions: J.T. Mey collected samples for a subset of the participants, generated and analysed the data, drafted and edited the manuscript; A. Hari, C.L. Axelrod, C.E. Fealy and M.L. Erickson collected samples for a subset of the participants, generated the data, and contributed to drafting and editing the manuscript; J.P. Kirwan and R.A. Dweik conceptualised the study, collected data, provided partial funding, and edited the manuscript. G.A. Heresi conceptualised the study, generated and analysed the data, provided funding, and edited the manuscript.

Conflict of interest: A. Hari has nothing to disclose. C.L. Axelrod has nothing to disclose. C.E. Fealy has nothing to disclose. M.L. Erickson has nothing to disclose. J.P. Kirwan reports grants from National Institutes of Health (UL1RR024989, U54GM104940), during the conduct of the study. R.A. Dweik reports grants from National Institutes of Health (R01HL130209), during the conduct of the study. G.A. Heresi reports grants from National Institutes of Health (K23HL125697), during the conduct of the study. J.T. Mey reports grants from National Institutes of Health (T32AT004094), during the conduct of the study. 
Support statement: This research was supported in part by the following grants: K23HL125697 (G.A. Heresi), R01HL130209 (R.A. Dweik), UL1RR024989, U54GM104940 (J.P. Kirwan) and T32AT004094 (J.T. Mey - trainee). Funding information for this article has been deposited with the Crossref Funder Registry.

\section{References}

1 Humbert M, Sitbon O, Yaici A, et al. Survival in incident and prevalent cohorts of patients with pulmonary arterial hypertension. Eur Respir J 2010; 36: 549-555.

2 Farber HW, Miller DP, Poms AD, et al. Five-year outcomes of patients enrolled in the REVEAL registry. Chest 2015; 148: 1043-1054.

3 Humbert M, Guignabert C, Bonnet S, et al. Pathology and pathobiology of pulmonary hypertension: state of the art and research perspectives. Eur Respir J 2019; 53: 1801887.

4 Badesch DB, Raskob GE, Elliott CG, et al. Pulmonary arterial hypertension: baseline characteristics from the REVEAL Registry. Chest 2010; 137: 376-387.

5 Ling Y, Johnson MK, Kiely DG, et al. Changing demographics, epidemiology, and survival of incident pulmonary arterial hypertension: results from the pulmonary hypertension registry of the United Kingdom and Ireland. Am J Respir Crit Care Med 2012; 186: 790-796.

6 Harvey LD, Chan SY. Emerging metabolic therapies in pulmonary arterial hypertension. J Clin Med 2017; 6: E43.

7 Heresi GA, Aytekin M, Newman J, et al. Plasma levels of high-density lipoprotein cholesterol and outcomes in pulmonary arterial hypertension. Am J Respir Crit Care Med 2010; 182: 661-668.

8 Barnes JW, Tian L, Heresi GA, et al. O-linked beta-N-acetylglucosamine transferase directs cell proliferation in idiopathic pulmonary arterial hypertension. Circulation 2015; 131: 1260-1268.

9 Heresi GA, Malin SK, Barnes JW, et al. Abnormal glucose metabolism and high-energy expenditure in idiopathic pulmonary arterial hypertension. Ann Am Thorac Soc 2017; 14: 190-199.

10 Lin T, Gu J, Huang C, et al. (1)H NMR-based analysis of serum metabolites in monocrotaline-induced pulmonary arterial hypertensive rats. Dis Markers 2016; 2016: 5803031.

11 Heresi GA, Mey JT, Bartholomew JR, et al. EXPRESS: plasma metabolomic profile in chronic thromboembolic pulmonary hypertension. Pulm Circ 2019; 10: 2045894019890553.

12 Aubert G, Martin OJ, Horton JL, et al. The failing heart relies on ketone bodies as a fuel. Circulation 2016; 133: 698-705.

13 Hemnes AR, Luther JM, Rhodes CJ, et al. Human PAH is characterized by a pattern of lipid-related insulin resistance. JCI Insight 2019; 4: 123611.

14 Haus JM, Solomon TP, Marchetti CM, et al. Free fatty acid-induced hepatic insulin resistance is attenuated following lifestyle intervention in obese individuals with impaired glucose tolerance. J Clin Endocrinol Metab 2010; 95: 323-327.

15 Kirwan JP, Kohrt WM, Wojta DM, et al. Endurance exercise training reduces glucose-stimulated insulin levels in 60- to 70-year-old men and women. J Gerontol 1993; 48: M84-M90.

16 DeFronzo RA, Tobin JD, Andres R. Glucose clamp technique: a method for quantifying insulin secretion and resistance. Am J Physiol 1979; 237: E214-E223.

17 Frigolet ME, Gutierrez-Aguilar R. The role of the novel lipokine palmitoleic acid in health and disease. Adv Nutr 2017; 8: 173S-181S.

18 Mittendorfer B, Liem O, Patterson BW, et al. What does the measurement of whole-body fatty acid rate of appearance in plasma by using a fatty acid tracer really mean? Diabetes 2003; 52: 1641-1648.

19 Vice E, Privette JD, Hickner RC, et al. Ketone body metabolism in lean and obese women. Metab Clin Exp 2005; 54: $1542-1545$

20 Elia M, Stubbs RJ, Henry CJ. Differences in fat, carbohydrate, and protein metabolism between lean and obese subjects undergoing total starvation. Obes Res 1999; 7: 597-604.

21 Polonsky KS, Given BD, Hirsch L, et al. Quantitative study of insulin secretion and clearance in normal and obese subjects. J Clin Invest 1988; 81: 435-441.

22 Cobelli C, Toffolo GM, Dalla Man C, et al. Assessment of beta-cell function in humans, simultaneously with insulin sensitivity and hepatic extraction, from intravenous and oral glucose tests. Am J Physiol Endocrinol Metab 2007; 293: E1-E15.

23 Maack C, Lehrke M, Backs J, et al. Heart failure and diabetes: metabolic alterations and therapeutic interventions: a state-of-the-art review from the Translational Research Committee of the Heart Failure Association-European Society of Cardiology. Eur Heart J 2018; 39: 4243-4254.

24 Moller JB, Dalla Man C, Overgaard RV, et al. Ethnic differences in insulin sensitivity, beta-cell function, and hepatic extraction between Japanese and Caucasians: a minimal model analysis. J Clin Endocrinol Metab 2014; 99: 4273-4280.

25 Nosadini R, Avogaro A, Trevisan R, et al. Acetoacetate and 3-hydroxybutyrate kinetics in obese and insulindependent diabetic humans. Am J Physiol 1985; 248: R611-R620.

26 Puchalska P, Crawford PA. Multi-dimensional roles of ketone bodies in fuel metabolism, signaling, and therapeutics. Cell Metab 2017; 25: 262-284.

27 Horton JL, Davidson MT, Kurishima C, et al. The failing heart utilizes 3-hydroxybutyrate as a metabolic stress defense. JCI Insight 2019; 4: 124079.

28 Lestan B, Walden K, Schmaltz S, et al. Beta-hydroxybutyrate decreases adenosine triphosphate degradation products in human subjects. J Lab Clin Med 1994; 124: 199-209.

29 Hevener AL, Bergman RN, Donovan CM. Novel glucosensor for hypoglycemic detection localized to the portal vein. Diabetes 1997; 46: 1521-1525.

30 d'Avignon DA, Puchalska P, Ercal B, et al. Hepatic ketogenic insufficiency reprograms hepatic glycogen metabolism and the lipidome. JCI Insight 2018; 3: 99762.

31 Veech RL, Bradshaw PC, Clarke K, et al. Ketone bodies mimic the life span extending properties of caloric restriction. IUBMB Life 2017; 69: 305-314.

32 Azevedo Jr. JL, Carey JO, Pories WJ, et al. Hypoxia stimulates glucose transport in insulin-resistant human skeletal muscle. Diabetes 1995; 44: 695-698. 
33 Thomas A, Belaidi E, Moulin S, et al. Chronic intermittent hypoxia impairs insulin sensitivity but improves whole-body glucose tolerance by activating skeletal muscle AMPK. Diabetes 2017; 66: 2942-2951.

34 Utzschneider KM, Kahn SE, Polidori DC. Hepatic insulin extraction in NAFLD is related to insulin resistance rather than liver fat content. J Clin Endocrinol Metab 2019; 104: 1855-1865.

35 Karpe F, Dickmann JR, Frayn KN. Fatty acids, obesity, and insulin resistance: time for a reevaluation. Diabetes 2011; 60: 2441-2449.

36 Bergman BC, Cornier MA, Horton TJ, et al. Effects of fasting on insulin action and glucose kinetics in lean and obese men and women. Am J Physiol Endocrinol Metab 2007; 293: E1103-E1111.

37 Sanders TAB, ed. Functional Dietary Lipids: Food Formulation, Consumer Issues and Innovation for Health. Cambridge, Woodhead Publishing, 2016. p. 332.

38 Tamaki M, Fujitani Y, Hara A, et al. The diabetes-susceptible gene SLC30A8/ZnT8 regulates hepatic insulin clearance. J Clin Invest 2013; 123: 4513-4524.

39 Piccinini F, Polidori DC, Gower BA, et al. Hepatic but not extrahepatic insulin clearance is lower in African American than in European American women. Diabetes 2017; 66: 2564-2570.

40 Hadengue A, Benhayoun MK, Lebrec D, et al. Pulmonary hypertension complicating portal hypertension: prevalence and relation to splanchnic hemodynamics. Gastroenterology 1991; 100: 520-528.

41 Jensen KJ, Alpini G, Glaser S. Hepatic nervous system and neurobiology of the liver. Compr Physiol 2013; 3 : 655-665.

42 Osei K, Gaillard T, Schuster D. Thiazolidinediones increase hepatic insulin extraction in African Americans with impaired glucose tolerance and type 2 diabetes mellitus. A pilot study of rosiglitazone. Metab Clin Exp 2007; 56: $24-29$.

43 Melenovsky V, Benes J, Franekova J, et al. Glucose homeostasis, pancreatic endocrine function, and outcomes in advanced heart failure. J Am Heart Assoc 2017; 6: e005290.

44 Home P. Cardiovascular outcome trials of glucose-lowering medications: an update. Diabetologia 2019; 62: 357-369.

45 Bril F, Barb D, Portillo-Sanchez P, et al. Metabolic and histological implications of intrahepatic triglyceride content in nonalcoholic fatty liver disease. Hepatology 2017; 65: 1132-1144.

46 Bril F, Lomonaco R, Orsak B, et al. Relationship between disease severity, hyperinsulinemia, and impaired insulin clearance in patients with nonalcoholic steatohepatitis. Hepatology 2014; 59: 2178-2187.

47 Polonsky KS, Rubenstein AH. C-peptide as a measure of the secretion and hepatic extraction of insulin. Pitfalls and limitations. Diabetes 1984; 33: 486-494.

48 Polonsky K, Frank B, Pugh W, et al. The limitations to and valid use of C-peptide as a marker of the secretion of insulin. Diabetes 1986; 35: 379-386.

49 De Bernardi Rodrigues AM, da Silva Cde C, Vasques AC, et al. Association of sleep deprivation with reduction in insulin sensitivity as assessed by the hyperglycemic clamp technique in adolescents. JAMA Pediatr 2016; 170: 487-494.

50 Shah SS, Ramirez CE, Powers AC, et al. Hyperglycemic clamp-derived disposition index is negatively associated with metabolic syndrome severity in obese subjects. Metab Clin Exp 2016; 65: 835-842. 